\title{
SINGULAR SETS OF SOME KLEINIAN GROUPS
}

\author{
TOHRU AKAZA
}

\section{Introduction}

In our paper [1] we showed that there exist Schottky groups whose singular sets have positive 1-dimensional measure. Since the example was very complicated, it is natural to seek for simpler examples. Further the problem how about the singular sets of more general groups occurs.

In $\S \S 1-3$ we investigate the measures of the singular sets of some Kleinian groups and the convergence problem of the $(-2)$-dimensional Poincare thetaseries. The main result is that there exist Kleinian groups whose fundamental domains are bounded by five mutually disjoint circles and whose singular sets have positive 1-dimensional measure. But it seems still open whether the singular sets of the Kleinian groups whose fundamental domains are bounded by four mutually disjoint circles can have positive 1-dimensional measure or not. In $\S 4$, as applications of the preceding chapters, similar problems about Schottky subgroups formed from Kleinian groups by inversion method are treated.

\section{§1. Kleinian groups whose fundamental domains are bounded by $\boldsymbol{N}$ mutually disjoint circles}

1. Consider the properly discontinuous groups $G$ of the linear transformations whose fundamental domain $B_{0}$ is bounded by $N$ mutually disjoint circles $\left\{K_{i}\right\}_{i=1}^{N}$. Then there exist two different kinds of generators. A generator $S_{i_{0}}$ of the first kind transforms the outside of a boundary circle $K_{i_{0}}$ onto the inside of a boundary circle $K_{i_{0}}^{\prime}$ different from $K_{i_{0}}$ and a generator $S_{j_{0}}$ of the second kind transforms the outside of $K_{j_{0}}$ onto the inside of $K_{j_{0}}$ itself. The former is the hyperbolic or loxodromic transformation and the latter is the elliptic trans formation with period 2 .

Take any generator of $G$

Received July 30, 1965. 


$$
T_{i}(z)=\frac{a_{i} z+b_{i}}{c_{i} z+d_{i}}, \quad\left(a_{i} d_{i}-b_{i} c_{i}=1\right) .
$$

If $T_{i}(z)$ is hyperbolic or loxodromic, then $a_{i}+d_{i}$ is either real and $\left|a_{i}+d_{i}\right|>2$ or complex and non zero. If $T_{i}(z)$ is the elliptic transformation with period 2 , then $T_{i}^{2}(z)$ is the identical transformation, so $a_{i}+d_{i}$ is zero.

2. Now we start from $B_{0}$ and form a properly discontinuous group of linear transformations with the fundamental domain $B_{0}$.

Take $2 p(N \geqq 2 p)$ boundary circles $\left\{H_{i}, H_{i}^{\prime}\right\}_{i=1}^{p}$ from $\left\{K_{i}\right\}_{i=1}^{N}$. Let $S_{i}$ be a hyperbolic or loxodromic generator which transforms the outside of $H_{i}$ onto the inside of $H_{i}^{\prime}$. We denote by $S_{i}^{-1}$ the inverse transformation of $S_{i}$. Then $\left\{S_{i}\right\}_{i=1}^{p}$ generate a Schottky group $G_{1}$, which is a subgroup of $G$ and whose fundamental domain $B_{1} \supset B_{0}$ is bounded by $\left\{H_{i}, H_{i}^{\prime}\right\}_{i=1}^{p_{1}}$. Let $\left\{T_{j}\right\}_{j=1}^{q}$ be the elliptic transformations with period 2 corresponding to the remaining boundary circles $\left\{K_{j}\right\}_{j=1}^{q}$, where $N-2 p=q$. Then $\left\{T_{j}\right\}_{j=1}^{q}$ generate a properly discontinuous group $G_{2}$ whose fundamental domain $B_{2} \supset B_{0}$ is the outside of the boundary circles $\left\{K_{j}\right\}_{j=1}^{q}$. By combining two groups $G_{1}$ and $G_{2}$, a new group $G_{1} \cdot G_{2}$, which is generated by $\left\{S_{i}\right\}_{i=1}^{p}$ and $\left\{T_{j}\right\}_{j=1}^{q}$, is obtained and is called a Kleinian group. It is easily seen that the fundamental domain of $G$ coincides with $B_{0}$ $=B_{1} \cap B_{2}$ and $G$ is properly discontinuous. In the special case of $N=2 p$, $G=G_{1}$ is a Schottky group, and if $N$ is odd, there exists necessarily at least one elliptic transformation with period 2 and $G$ is a Kleinian group. If $p=0$ especially, $G$ is generated by the only elliptic transformations $\left\{T_{j}\right\}_{j=1}^{N}$ with period 2. To the domain $B_{0}$ with $N$ boundary circles, there exist

$$
N ! \sum_{p=1}^{[N / 2]} \frac{1}{2^{p} \cdot p !(N-2 p) !}
$$

Kleinian groups in all, according to determination of generators, where $\left[\frac{N}{2}\right]$ denotes the maximal integer not exceeding $\frac{N}{2}$.

3. Let $G$ be a Kleinian group generated by combining two groups $G_{1}$ and $G_{2}$ as the above, where $G_{1}$ is a Schottky group with fundamental domain $B_{1}$ generated by $p$ generators and their inverses and $G_{2}$ with fundamental domain $B_{2}$ is also generated by $q=N-2 p$ elliptic transformations with period 2 and of course the fundamental domain of $G$ is $B_{0}=B_{1} \cap B_{2}$. 
We denote by $S T$ the transformation obtained by composition of transformations $S$ and $T$ contained in $G$, that is,

$$
S T(z)=S(T(z)) .
$$

We put $S S=S^{2}$ and $S^{\lambda}=S \cdot S^{\lambda-1}$ inductively for any integer $\lambda(>1)$. For a negative integer $\lambda, S^{\lambda}$ denotes $\left(S^{-1}\right)^{|\lambda|}$. Then any element $S$ of $G$ has the form

$$
\begin{aligned}
& S=S_{\left(\lambda_{k}\right)} T_{j_{k}} \cdots S_{\left(v_{1}\right)} T_{j_{1}} S_{\left(v_{0}\right)}, \text { viz., } \\
& S(z)=S_{\left(v_{k}\right)}\left(T _ { j _ { k } } \left(\cdots\left(T_{j_{1}}\left(S_{\left(v_{0}\right)}(z)\right) \cdots\right),\right.\right.
\end{aligned}
$$

where $\nu_{i}(i=0, \ldots, k)$ are integers and $S_{\left(\nu_{i}\right)}$ denotes the $\left|\nu_{i}\right|$ product of generators of $G_{1}$ or their inverses and $T_{j_{i}}\left(T_{j_{i}}^{2}=\right.$ identity) denotes the generator of $G_{2}$. We call the sum

$$
m=\sum_{i=0}^{k}\left|\nu_{i}\right|+k
$$

the grade of $S$.

The image $S\left(B_{0}\right)$ of the fundamental domain $B_{0}$ by $S(\in G)$ with grade $m$ $(\neq 0)$ is bounded by $N$ circles $S\left(H_{i}\right), S\left(H_{i}^{\prime}\right)$ and $S\left(K_{j}\right),(i=1, \ldots, p, j=1, \ldots$, $q, N=2 p+q)$, the one $C^{(m-1)}$ of which is contained in the boundary of the image of $B_{0}$ under some $T(\in G)$ with grade $m-1$. For simplicity, we say that the outer boundary circle $C^{(m-1)}$ of $S\left(B_{0}\right)$ is a circle of grade $m$. Circles $\left\{H_{i}, H_{i}^{\prime}\right\}_{i=1}^{p} \cup\left\{K_{j}\right\}_{j=1}^{q}$, which bound $B_{0}$, are of grade 1 . The number of circles of grade $m$ is obviously equal to $N(N-1)^{m-1}$.

Denote by $D_{m}$ the $N(N-1)^{m-1}$-ply connected domain bounded by the whole circles of grade $m$. Evidently $\left\{D_{m}\right\}(m=1,2, \ldots)$ is a monotone increasing sequence of domains. The complementary set $D_{m}^{c}$ of $D_{m}$ with respect to the extended $z$-plane consists of $N(N-1)^{m-1}$ mutually disjoint closed discs. The set $E=\bigcap_{m=1}^{\infty} D_{m}^{c}$ is perfect and nowhere dense. We call $E$ the singular set of $G$. The group $G$ is properly discontinuous in the complementary set of $E$. It is well-known that, in the special case when $G$ is a Schottky group with $p \geqq 2$, the logarithmic capacity of $E$ is positive (See Myrberg [4]) and that the 2-dimensional measure of $E$ is equal to zero (See Sario [6]). Applying Myrberg's method to such a Kleinian group, it is seen that the logarithmic capacity of $E$ of this group is positive in the case of $N \geqq 3$. In our paper [1], we proved, using the example of the fundamental domain of such a group bounded by 36 circles, that there exist Schottky groups whose singular sets have the 
positive 1-dimensional measure.

4. Let $H(z)$ be a rational function none of whose poles is contained in the singular set $E$ of the Kleinian group $G$. Denote by $z_{j}=\left(a_{j} z+b_{j}\right) /\left(c_{j} z+d_{j}\right)$ $(j=0,1, \ldots)$ all the elements of $G$. The identical transformation of $G$ is denoted by $z_{0}$.

Consider the series

$$
\Theta_{\nu}(z)=\sum_{j=0}^{\infty} H\left(z_{j}\right)\left(c_{j} z+d_{j}\right)^{-\nu},
$$

where $\nu$ is a positive integer. This is a so-called $(-\nu)$-dimensional Poincaré theta-series.

Let $D$ be the complementary domain of the set $E$ and $D^{\prime}$ be a relatively closed subdomain of $D$. Since the point $-d_{j} / c_{j}(j \neq 0)$ is the image of infinity by the inverse transformation $z_{j}^{-1}$ of $z_{j}(j \neq 0)$ and since $G$ is properly discontinuous in $D$, there are only finitely many points $-d_{j} / c_{j}(j \neq 0)$ in $D^{\prime}$. Denote by $D^{\prime \prime}$ a non-empty subdomain of $D^{\prime}$ obtained by deleting suitable neighborhoods of points $-d_{j} / c_{j}$ and $\infty$.

Let $e_{i}(i=1, \ldots, k)$ be poles of $H(z)$ in $D$ and let $U_{i}$ be neighborhoods of $e_{i}$ such that $|H(z)|=M_{1}$ on the boundary of $U_{i}$. By using the proper discontinuity of $G$ and by taking $M_{1}$ sufficiently large, we may assume that $D^{*}=D^{\prime \prime}$ $-\bigcup_{S \in G} \bigcup_{i=1}^{k} S\left(U_{i}\right)$ are not empty.

Then we have the following propositions (cf. [1]).

Proposition 1. The $(-\nu)$-dimensional Poincare theta-series

$$
\Theta_{\nu}(z)=\sum_{j=0}^{\infty} H\left(z_{j}\right)\left(c_{j} z+d_{j}\right)^{-\nu}, z_{j}=\frac{a_{j} z+b_{j}}{c_{j} z+d_{j}} \in G
$$

converges absolutely and uniformly in $D^{*}$ if and only if the series

$$
\sum_{j=0}^{\infty}\left(c_{j} z+d_{j}\right)^{-\imath}
$$

converges absolutely and uniformly in $D^{\prime \prime}$.

We put

$$
P_{\nu}(z)=\sum_{j=0}^{\infty}\left|c_{j} z+d_{j}\right|^{-v}
$$

where $\nu$ is a positive number. We call $P_{\nu}(z)$ the $(-\nu)$-dimensional $P$-series. 
Petersson [5] showed that if $G$ is a Fuchsian group, $P_{\nu}(z)$ converges for $\nu>2$, and that if $G$ is a Fuchsian group of the first kind, $P_{\nu}(z)$ diverges for $\nu<2$. We have the following

Proposition 2. The series $P_{\nu}(z)$ converges uniformly in $D^{\prime \prime}$ if and only if the series

$$
\sum_{j=1}^{\infty}\left|c_{j}\right|^{-\nu}, \quad(\nu>0)
$$

converges.

Let

$$
S^{(m)}: z^{\prime}=S^{(m)}(z)=\frac{a z+b}{c z+d}, \quad(a d-b c=1)
$$

be a transformation of grade $m(\geqq 1)$ in $G$. Then the radius $r_{0}$ of a circle $C$ of grade $m$ by $S^{(m)}(z)$ is given by

$$
2 \pi r_{0}=\int_{B}\left|\frac{d S^{(m)}(z)}{d z}\right||d z|=\int_{H} \frac{|d z|}{|c z+d|^{2}}
$$

where $H$ is a suitable one in $\left\{H_{i}, H_{i}^{\prime}\right\}_{i=1}^{p} \cup\left\{K_{j}\right\}_{j=1}^{q}$ which $S^{(m)}$ carries to $C$. Hence, we have

$$
2 \pi r_{0}=\frac{1}{|c|^{2}} \int_{H} \frac{|d z|}{|z+(d / c)|^{2}}
$$

Again we note that the point $-d / c$ is outside of $B_{0}$. If we put

$$
\Delta=\max _{z \in H}|z+(d / c)| \text { and } \delta=\min _{z \in H}|z+(d / c)| \text {, }
$$

then

$$
\frac{r}{\Delta^{2}} \cdot \frac{1}{|c|^{2}} \leqq r_{0} \leqq \frac{r}{\delta^{2}} \cdot \frac{1}{|c|^{2}} .
$$

where $r$ is the radius of $H$.

Such inequality holds for all circles of grade $m$. Hence we have the following.

Proposition 3. The series $\sum_{j=1}^{\infty}\left|c_{j}\right|^{-\nu}$ converges if and only if $\sum_{m=1}^{\infty} l_{m}^{(\nu)}$ converges, where $l_{m}^{(\nu)}$ is the sum of terms $\left(r^{(m)}\right)^{v / 2}$ obtained for radii $r^{(m)}$ of circles of grade $m$.

Combining Propositions 1; 2 and 3, we have the following important Proposition 4 , which we shall use later. 
Proposition 4. Let $\nu$ be a positive integer. The following four propositions are equivalent to each other: (i) The $(-\nu)$-dimensional Poincare theta-series $\Theta_{\nu}(z)$ converges absolutely and uniformly in $D^{*}$. (ii) The $(-\nu)$-dimensional P-series $P_{\nu}(z)$ converges uniformly in $D^{\prime \prime}$. (iii) The series $\sum_{j=1}^{\infty}\left|c_{j}\right|^{-\nu}$ converges. (vi) The series $\sum_{m=1}^{\infty} l_{m}^{(1)}$ converges.

It is evident that, if $\lim _{m \rightarrow \infty} l_{m}^{(\nu)}=0$, then the singular set of $G$ is of $\left(\frac{\nu}{2}\right)$ dimensional measure zero. Hence, from Proposition 4, we get

Proposition 5. If any one of the conditions (i), (ii), (iii) and (iv) in Proposition 4 is valid, then the singular set of $G$ is of $\left(\frac{\nu}{2}\right)$-dimensional measure zero.

5. We shall state the concept of isometric circles of linear transformations due to Ford [2] and some important properties of them.

For a linear transformation of the form

$$
T(z)=\frac{a z+b}{c z+d}, \quad a d-b c=1, c \neq 0,
$$

the circle $I:|c z+d|=1$ is called the isometric circle of the transformation. The radius of $I$ equals $1 /|c|$.

(I) By a transformation lengths and areas inside its isometric circle are increased in magnitude and lengths and areas outside the isometric circle are decreased in magnitude. A transformation carries its isometric circle into the isometric circle of the inverse transformation. The radii of the isometric circles of a transformation and its inverse are equal.

Let $G$ be a properly discontinuous group of linear transformations. We suppose that, if an element of $G$ transforms the point at infinity into itself, then the element is the identity of $G$. Consider two arbitrary transormations of $G$

$$
T: T(z)=\frac{a z+b}{c z+d}, \quad a d-b c=1, c \neq 0,
$$

and

$$
S: S(z)=\frac{\alpha z+\beta}{\gamma z+\delta}, \alpha \delta-\beta \gamma=1, \gamma \neq 0 .
$$

For a moment we assume that $S \neq T^{-1}$. The isometric circle of $S T=S(T(z))$ is the circle 


$$
|(\gamma a+\delta c) z+\gamma b+\delta d|=1
$$

Denote by $I_{S}, I_{s}^{\prime}, I_{T}, I_{T}^{\prime}$ and $I_{S T}$ isometric circles of $S, S^{-1}, T, T^{-1}$ and $S T$, respectively. Let $g_{S}, g_{s}^{\prime}, g_{T}, g_{T}^{\prime}$ and $g_{S T}$ be their centers, and let $R_{S}, R_{T}$ and $R_{S T}$ be radii of $I_{S}, I_{T}$ and $I_{S T}$.

As to these values, the relation

$$
R_{S T}=\frac{1}{|\gamma a+\delta c|}=\frac{R_{S} \cdot R_{T}}{\left|g_{T}^{\prime}-g_{s}\right|}
$$

holds.

As to the location of isometric circles, we have from (I) the following.

(II) If $I_{S}$ and $I_{T}^{\prime}$ are exterior to each other, then $I_{S T}$ is contained in $I_{T}$. If $I_{S}$ and $I_{T}^{\prime}$ are tangent externally, then $I_{S T}$ lies in $I_{T}$ and is tangent internally.

If the grade of the transformation in $G$ is $m$, its isometric circle is called an isometric circle of grade $m$. The number of the isometric circles with grade $m$ is obviously equal to $N(N-1)^{m-1}$.

From the definition of isometric circles follows:

Proposition 6. The convergence of the series $\sum_{j=1}^{\infty}\left|c_{j}\right|^{-v}$ is equivalent to the convergence of the sum of $\boldsymbol{\nu}$-th powers of radii of isometric circles for all the elements of $G$.

\section{§2. Measure of the singular sets of Kleinian groups}

6. Given a set $\varepsilon$ of points in the $z$-plane and a positive number $\delta$, we denote by $I(\delta, \varepsilon)$ a family of a countable number of closed discs $U$ of diameter $l_{0} \leqq \delta$ such that every point of $\varepsilon$ is an interior point of at least one $U$.

We call the quantity

$$
\Lambda^{\eta} \varepsilon=\lim _{\delta \rightarrow 0}\left[\inf _{(I(\delta, \varepsilon)\}} \sum_{l \in L(\delta, \varepsilon)} l_{\varepsilon}^{\eta}\right]
$$

the $\eta$-dimensional measure of $\varepsilon$.

7. Denoting by $r_{j}^{(m)}$ and by $r_{i}^{(m+1)}(i=1, \ldots, N-1)$ the radius of the outer boundary circle $C_{j}^{(m)}$, that is, a circle of grade $m$ and the radii of $N-1$ inner boundary circles $C_{i}^{(m+1)}(i=1, \ldots, N-1)$ of the image $B_{m}$ of the fundamental domain $B_{0}$ by a transformation $S^{(m)}(\in G)$ with grade $m$, we have the following (See [1].), 
PROPOSITION 7. There exist positve constants $K_{0}(<1)$ and $k_{0}$ depending only on $B_{0}$ such that

$$
k_{0} r_{j}^{(m)} \leqq r_{i}^{(m+1)} \leqq K_{0} r_{j}^{(m)} . \quad(i=1, \ldots, N-1) .
$$

8. In [1] we obtained the important criterion which determines that the singular set $E$ of a Schottky group $G$ has the positive $\eta$-dimensional measure. Since the method will be needed in the following, we state it here again.

Denote by $F_{n_{0}}$ the family of all closed discs bounded by circles of grade $n$ $\left(\geqq n_{0}\right)$. It is easy to see that $F_{n_{0}}$ is a covering of the singular set of our Kleinian group $G$ and that the diameter of any disc of $F_{n_{0}}$ is less than a given. $\delta(>0)$ for sufficiently large $n_{0}$. This fact is verified by Proposition 7 .

Consider a family $I(\delta, E)$ of coverings of $E$ stated in No. 6 . Since $E$ is compact, the set $E$ is covered by a finite number of discs $\mathbb{E}_{1}, \ldots, \mathbb{E}_{k}$ of a covering of $E$ in $I(\delta, E)$. Take an arbitrary $\mathscr{E}_{i}$ among these $k$ circles and let $l_{i}(\leqq \delta / 2)$ be the radius of $E_{i}$.

Let $\delta$ be sufficiently small. For a $E_{i}$ fixed, we can find closed $\operatorname{discs}{ }^{i} C^{\left(m_{1}\right)}$, $\ldots,{ }^{i} C^{\left(m_{\mathrm{N}(i)}\right)}$ in $\bigcup_{n=1}^{\infty} F_{n}$ satisfying the following conditions:

(i) The radius ${ }^{i} r^{\left(m_{j}\right)}$ of ${ }^{i} C^{\left(m_{j}\right)}(1 \leqq j \leqq N(i))$ of grade $m_{j}$ is larger than $l_{i}$;

(ii) There exist at least one circle of grade $m_{j}+1$ lying inside the boundary of ${ }^{i} C^{\left(m_{j}\right)}$, meeting $\varepsilon_{i}$ and of radius ${ }^{i} r^{\left(m_{j}+1\right)}$ not greater than $l_{i}$;

(iii) $\bigcup_{j=1}^{i} C^{\left(m_{j}\right)} \supset \xi_{i} \cap E$.

It is easy to see that there exist a constant $\kappa$ independent of $i$ such that $N(i) \leqq \kappa$. We can prove $\kappa=5$ by some geometrical consideration.

By the inequality (6) of Proposition 7,

$$
k_{0}{ }^{i} r^{\left(m_{j}\right)} \leqq{ }^{i} r^{\left(m_{j}+1\right)} \leqq l_{i}<{ }^{i} r^{\left(m_{j}\right)} .
$$

Construct such discs $\left\{{ }^{i} C^{\left(m_{j}\right)}\right\}$ for every $\mathfrak{E}_{i}(i=1, \ldots, k)$. Then it is obvious that $\bigcup_{i=1}^{k} \bigcup_{j=1}^{N(i)} C^{\left(m_{j}\right)} \supset E$ and

$$
\sum_{i=1}^{k} \sum_{j=1}^{N(i)}\left({ }^{i} r^{\left(m_{j}\right)}\right)^{\eta} \leqq \kappa k_{0}^{-\eta} \sum_{i=1}^{k} l_{i}^{\eta}
$$

Thus we have

Proposition 8. Let $F_{n_{0}}^{\delta / k_{0}}$ be a covering of $E$ constructed by discs in $F_{n_{0}}$ whose radii are not greater than $\delta / 2 k_{0}$ and let $r_{c}$ be the radius of a disc $C$ in $F_{n_{0}}^{\delta / k_{0}}$. Then it holds 


$$
L^{\eta} E=\lim _{\delta \rightarrow 0} \inf _{\left(r_{n_{0}}^{\delta / k_{0}}\right)} \sum_{c \in F_{n_{0}}^{\delta / k_{0}}}\left(2 r_{c}\right)^{\eta} \leqq \kappa\left(\frac{k_{0}}{2}\right)^{-\eta} \Lambda^{\eta} E,
$$

By Proposition 8 , we can prove

Proposition 9. Given a Kleinian group defined in $\S 1$, if

$$
\sum_{T_{i}}\left(R_{S(m)}\right)^{\mu} \geqq\left(R_{S(m-1)}\right)^{\mu}, \quad\left(0<\mu<4, S^{(m)}=S^{(m-1)} T_{i}\right)
$$

for radius $R_{S^{(m-1)}}$ of any isometric circle $I_{S^{(m-1)}}$ of grade $m-1$ and radii $R_{S^{(m)}}$ of $N-1$ isometric circles $I_{s^{(m}}$. of grade $m$, then the $\left(\frac{\mu}{2}\right)$-dimensional measure of the singular set $E$ of $G$ is positive.

Proof. Take a covering $F_{n_{0}}^{\delta / k_{0}}$ of $E$ constructed by a finite number of close discs $D_{S\left(m_{1}\right)}^{1}, \ldots, D_{S\left(m_{Q}\right)}^{1}$, which are bounded by circles

$$
C_{S^{\left(m_{1}\right)}}^{1}, \ldots, C_{S^{(}\left(m_{Q}\right)}^{1}
$$

respectively, where $C_{S\left(m_{j}\right)}^{1}(1 \leqq j \leqq Q)$ is a circle of grade $m_{j}$, that is, an outer boundary circle of the image $S^{\left(m_{j}\right)}\left(B_{0}\right)$.

Denote by $r_{S}\left(m_{j}\right)$ the radius of a circle $C_{s}^{1}\left(m_{j}\right)$. Then, from (4)

$$
\sum_{j=1}^{Q}\left(r_{S^{\left(m_{j}\right)}}\right)^{\mu / 2} \geqq k(G) \sum_{j=1}^{Q}\left(R_{\mathcal{S}^{\left(m_{j}\right)}}\right)^{\mu},
$$

where $R_{S^{(}\left(m_{j}\right)}$ is the radius of the isometric circle $I_{S^{(m)}}$.

From the construction of $F_{n_{0}}^{\delta / k_{0}}$, there exist in (9) some systems $\left\{W_{m_{k^{*}}}^{1}\right.$, each of which consists of $N-1$ boundary circles with the following properties :

(i) $N-1$ circles of $W_{m_{k^{*}}}^{1}$ have same grade number $m_{k}^{*}$, while the grade of circles of different systems are not necessarily equal,

(ii) $N-1$ circles of each system $W_{m_{k^{*}}}^{1}$ are bounded by a circle of grade $m_{k}^{*}-1$.

Let $C_{s_{i}\left(m_{k^{*}}\right)}^{1}(i=1,2, \ldots, N-1)$ and $C_{s^{\left(m_{k^{*}}-1\right)}}$ be circles of a system $W_{m_{k^{*}}}^{1}$ and a circle surrounding them respectively, where $S_{i}^{\left(m_{k^{*}}\right)}=S^{\left(m_{\left.k^{*}-1\right)}\right.} T_{i}(i=1,2$, $\ldots, N-1 ; T_{i}$; a generator or its inverse). By the assumption (8), it holds, for each system,

$$
\sum_{i=1}^{N-1}\left(R_{S_{i}\left(m_{k}\right)}\right)^{\mu} \geqq R_{S}^{\eta}\left(m_{k^{*}}-1\right)
$$

After replacing $N-1$ circles of each system $W_{m_{k}}^{1}$ by a circle surrounding 
them, we have also a new covering of $E$ consisting of closed discs $D_{s^{\left(m_{1}\right)}}^{2}, \ldots$, $D_{S}^{2}\left(m Q^{\prime}\right)$, which are bounded by circles

$$
C_{S\left(m_{1}\right)}^{2}, \ldots, C_{\left.s^{m} Q^{\prime}\right),}^{2} \quad\left(Q^{\prime}<Q\right)
$$

Then there exist in (11) some systems $\left\{W_{m l^{*}}^{2}\right\}$ which satisfy the above condition (i) and (ii) and hence, for each system of $\left\{W_{m l^{*}}^{2}\right\}$, it holds also

$$
\sum_{i=1}^{N-1}\left(R_{S_{i}\left(m l^{*}\right)}\right)^{\mu} \geqq R_{S}^{\mu}\left(m l^{*}-1\right)
$$

Repeating this procedure, we obtain the following

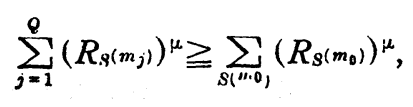

where $m_{0}=\min _{1 \leqq \equiv} m_{j}$ and the summation in the right hand side is taken over all transformations in $G$ with grade $m_{0}$. By a similar argument, we see

$$
\sum_{S\left(m^{\prime+0}\right)}\left(R_{S^{(}\left(m_{0}\right)}\right)^{\mu} \geqq \sum_{\left.S 1^{1}\right)}\left(R_{S^{(1)}}\right)^{\mu},
$$

where $\sum_{s^{(1)}}$ denotes the sum with respect to all generators and their inverses. Here the quantity in the right hand side is a positive constant. Thus, for any covering $F_{n_{0}}^{\delta / k_{0}}$ of $E$, we have from (10), (12) and (13)

$$
\sum_{j=1}^{Q}\left(r_{S^{(}\left(m_{j}\right)}\right)^{\mu / 2} \geqq k(G) \sum_{S^{(1)}}\left(R_{S^{(1)}}\right)^{\mu}>0
$$

Putting $\eta=\frac{\mu}{2}$ in (7), we can prove our proposition from the above inequality and Proposition 8.

Remark. In [1], we obtained the following result: given a Schottky group $G$, if

$$
\sum_{T_{i}^{\prime}}\left(R_{S^{(m)}}\right)^{\mu} \geqq R_{S}^{\mu}(m-1), \quad\left(0<\mu<4, S^{(m)}=T_{i} S^{(m-1)}\right),
$$

then the $\left(\frac{\mu}{2}\right)$-dimensional measure of the singular set $E$ of $G$ is positive.

This Theorem is valid for a Kleinian group defined in No. 2. But the process of proving Theorem contained the obscurity with respect to the relation between a covering consisting of the images of $B_{0}$ by transformations of $G$ and radii of their isometric circles.

From the property (II) in No. 5 , it is seen that $R_{\mathcal{S}^{(m-1)}}$ and $R_{\mathcal{S}^{(m)}}\left(S^{(m)}=\right.$ 
$T_{i} S^{(m-1)}$ are the radius of the isometric circle $I_{S^{(m-1)}}$ and the radii of $N-1$ isometric circles $I_{S^{(m)}}$ contained in $I_{S^{(m-1)}}$. By the property (I) in No. 5, (14) is equivalent to the condition

$$
\left.\sum_{T_{i}^{-1}}\left(R_{S^{-(m)}}\right) \geqq R_{S^{-(m-1)}}^{\mu}\right), \quad\left(S^{-(m)}=S^{-(m-1)} T_{i}^{-1}\right)
$$

and this is also equivalent to (8).

\section{§. Computing functions of a Kleinian group}

9. Let us consider a transformation

$$
S^{(m)}=S^{(m-1)} T_{1}=S^{(m-2)} T_{2} T_{1}, \quad\left(T_{2} \neq T_{1}^{-1}\right)
$$

of a Kleinian group $G$, where $T_{1}$ and $T_{2}$ are generators or their inverses. Let $R_{S^{(m)}}$ and $R_{S^{(m-1)}}$ be the radii of the isometric circles of $S^{(m)}$ and $S^{(m-1)}$. Then we have from (5)

$$
\left(\frac{R_{S^{(m)}}}{R_{S^{(m-1)}}}\right)^{\mu}=\frac{R_{T_{1}}^{\mu}}{\left|g_{T_{1}}^{\prime}-g_{S^{(m-1)}}\right|^{\mu}}, \quad(0<\mu<4) .
$$

We consider the following function

$$
f_{T_{2}}^{(\mu)}(z)=\sum_{T_{1}-1} \frac{R_{T_{1}}^{\mu}}{\left|g_{T_{1}-1}-z\right|^{\mu}}, \quad\left(T_{2} \neq T_{1}^{-1}\right)
$$

for the boundary circle $H_{i_{1}}$ of $B_{0}$, which is one of $B_{0}$ mapped onto $H_{i_{1}}^{\prime}$ by $T_{2}$, where $z$ varies on the closed disc bounded by $H_{i_{1}}$, and the notation $\sum_{T_{1}-1}$ denotes the summation with respect to the $N-1$ generators or their inverses except $T_{2}$. It $T_{2}$ is an elliptic transformation with period 2, then $H_{i_{1}}=H_{i_{1}}^{\prime}$.

It is obvious that

$$
f_{T_{2}}^{(\mu)}\left(g_{s^{(m-1)}}\right)=\sum_{T_{1}-1} \frac{R_{T_{1}}^{\mu}}{\left|g_{T_{1}-1}-g_{s^{(m-1)}}\right|^{\mu}} \cdot \quad\left(T_{2} \neq T_{1}^{-1}\right),
$$

where $g_{T_{1}-1}$ does not belong to the closed disc bounded by a boundary circle $H_{i_{1}}$ and $g_{s^{(m-1)}}$ is surrounded by $H_{i_{1}}$, since $g_{s(m-1)}=S^{-(m-1)}(\infty),\left(S^{-(m-1)}=\right.$ $\left.T_{2}^{-1} S^{-(m-2)}, \infty \in B_{0}\right)$ and $S^{-(m-1)}\left(B_{0}\right)$ is contained in the domain bounded by $H_{i_{1}}$. We call $f_{T_{2}}^{(\mu)}(z)$ the $\mu$-dimensional computing function of $T_{2}$ and there exist $N$ computing functions $f_{T_{\nu}}^{(\mu)}(z),(\nu=1, \ldots, N)$ in all, since the first element $T_{2}$ of $S^{(m-1)}$ is any generator or its inverse of $G$. Such functions $\left\{f_{T_{\nu}}^{(\mu)}(z)\right\} \quad(\nu=1$, $\ldots, N)$ are called the $\mu$-dimensional computing functions of a Kleinian group 
G.

10. Take a generator $T_{1}$ and its $\mu$-dimensional computing function

$$
f_{T_{2}}^{(\mu)}(z)=\sum_{T_{\nu} \neq T_{1}} \frac{R_{T_{\nu}}^{\mu}}{\left|g_{T_{\nu}}-z\right|^{\mu}},
$$

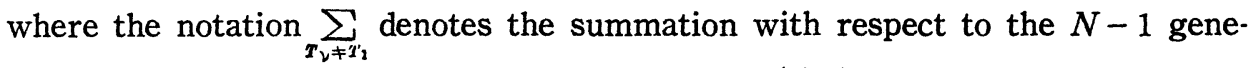
rators or their inverses $T_{\nu}$ except $T_{3}$. Then $f_{T_{1}}^{(\mu)}(z)$ is defined in the closed disc $D_{T_{1}}:\left|z-a_{t_{1}}\right| \leqq r_{t_{1}}$ bounded by $H_{t_{1}}$ which is a boundary circle of $B_{0}$ mapped onto $H_{t_{1}}^{\prime}$ by $T_{1}$. Since any center $g_{T_{\nu}}$ of the isometric circles of generators or their inverses $T_{\nu}\left(T_{\nu} \neq T_{1}\right)$ is a pole of $T_{\nu}$, it is in the outside of $D_{T_{1}}$ and bence the denominator of each term of $f_{T_{1}}^{(\mu)}(z)$ does not vanish. From this fact $f_{T_{1}}^{(\mu)}(z)$ is uniformly continuous in $D_{T_{1}}$. Then we can choose $\delta$ depending only on any small $\varepsilon$, so that it holds $\left|f_{T_{1}}^{(\mu)}(z)-f_{r_{1}}^{(\mu)}\left(z^{\prime}\right)\right|<\varepsilon$ for $z$ and $z^{\prime}$ satisfying $\left|z-z^{\prime}\right|<\delta$ in $D_{T_{1}}$. Denote by $E_{1}$ the subset of $E$ contained in $D_{T_{1}}$. Since, from Proposition 7 , any radius $r^{(m)}$ of circles of grade $m$ is equal or less than $K_{0}^{m-1} r^{(1)}\left(K_{0}<1\right)$, which tends to zero for $m \rightarrow \infty$, there exists a grade number $m_{0}$ depending only on $\delta$ so that for any $S^{(m)}=S^{(m-1)} T_{1}, m \geqq m_{0}$, there is $z_{0} \in E_{1}$ such that $g_{s^{(m)}} \in D_{\delta}$ $\left(z_{0}\right)$, where $D_{\delta}\left(z_{0}\right)$ denotes the disc with center $z_{0}$ and with radius $\delta$. Hence it can be seen that

$$
\left|f_{T_{1}}^{(\mu)}\left(z_{0}\right)-f_{T_{1}}^{(\mu)}\left(g_{\left.s^{(m)}\right)}\right)\right|<\varepsilon, \quad\left(\forall m \geqq m_{0}, S^{(m)}=S^{\left(m_{0}\right)} S^{\left(m^{\prime}\right)}\right) .
$$

Suppose that it bolds

$$
f_{T_{1}}^{(\mu)}(z)>\lambda_{1}, \quad \text { for any } z \in E_{1}
$$

Then we have from (16) and (17)

$$
\left|f_{T_{1}}^{(\mu)}\left(g_{S^{(m)}}\right)\right| \geqq f_{T_{1}}^{(\mu)}\left(z_{0}\right)|-| f_{Y_{1}}^{(\mu)}\left(g_{S^{(m)}}\right)-f_{i_{1}}^{(\mu)}\left(z_{0}\right) \mid>\lambda_{1}-\varepsilon .
$$

Now we have the following

Theorem 1. Let $G$ be a Kleinian group whose fundamental domain is bounded by $N$ boundary circles as in No. 2. If it holds that

$$
f_{i_{1}}^{(\mu)}(z)>\lambda_{i}>1, \quad(i=1, \ldots, N)
$$

on the singlar subset $E_{i}$ of $E$ contained in the boundary circle $H_{i}(i=1, \ldots, N)$ of $B_{0}$ respectively, then the singular set $E$ of $G$ has the positive $\left(\frac{\mu}{2}\right)$-dimensional measure. 
Proof. For any $i$, take $\varepsilon$ so small that it may hold $\lambda_{i}-\varepsilon>1(i=1, \ldots$, $N)$. Then we can determine the grade number $m_{0}$ such that the inequalities

$$
f_{T_{i}}^{(\mu)}\left(g_{S^{(m)}}\right)>\lambda_{i}-\varepsilon>1, \quad\left(m \geqq m_{0} ; i=1, \ldots, N\right)
$$

hold. Hence we have the following inequalities

$$
\sum_{T_{1}^{\prime}}\left(R_{S^{(m)}}\right)^{\mu} \geqq R_{S(m-1)}^{\mu}, \quad\left(S^{(m)}=S^{(m-1)} T_{1}=S^{(m-2)} T_{2} T_{1}, T_{2}^{-1} \neq T_{1}\right),
$$

for radius $R_{S^{(m-1)}}$ of any isometric circle $I_{S^{(m-1)}}$ of grade $m-1$ and radii $R_{S(m)}$ of the $N-1$ isometric circles $I_{s(m)}$ of grade $m$. Thus, by Proposition 9, we get the theorem. q.e.d.

Noting Proposition 4, we get the following

Corollary. If the condition (18) is satisfied for the computing functions of the Kleinian group, then the $(-\mu)$-dimensional P.series $P_{\mu}(z)$ does not converge in $D^{\prime \prime}$.

\section{§ 4. Examples of Kleinian groups whose singular sets have positive 1-dimensional measure}

11. P. J. Myrberg [3] treated also the convergence problem of the $(-2)$. dimensional Poincaré theta-series $\Theta_{2}(z)$ with respect to Schottky groups and Kleinian groups and gave the examples in which $\Theta_{2}(z)$ does not converge. But in his paper it was not treated from the view point of the measure of the singular sets of such groups.

In this chapter, by using the condition (for $\mu=2$ ) of Theorem 1, we shall give, more systematically, the examples in which the singular sets of Kleinian groups have positive 1-dimensional measure and $\Theta_{2}(z)$ does not converge in $D^{*}$. Further we shall try to make the number $N$ of the boundary circles as small as possible.

As the preliminary to give examples, we shall show how to construct a transformation $T$ which maps the outside of a circle $H$ onto the inside of another circle $H^{\prime}$, where $H$ and $H^{\prime}$ have equal radii, though in general we can set up infinitely many such transformations.

Denote two circles by

$$
H:|z-q|=r, H^{\prime}:\left|z-q^{\prime}\right|=r .
$$

It $T$ is restricted by the conditions : $q^{\prime}=T(\infty)$ and $q=T^{-1}(\infty)$, it is easily seen that $T$ has the following form 


$$
z^{\prime}=T(z)=\frac{q^{\prime} z-\left(q q^{\prime}+r^{2} e^{i \theta}\right)}{z-q}
$$

where $\theta$ is any real number and the isometric circles $I_{T}$ and $I_{T}^{\prime}$ are $H$ and $H^{\prime}$, respectively.

12. Example The case of $N=5$.

Consider the four circles $H_{j}(j=1,2,3,4)$ with centers $a_{j}=\sqrt{2} e^{i(2 j-1) \pi / 4}$ $\left(i^{2}=-1\right)$ and equal radii $R=1-\varepsilon$, respectively. If we let these four circles correspond to two hyperbolic transformations $S_{1}$ and $S_{2}$ by (19) such that the outside of $H_{1}$ is mapped onto the inside of $H_{3}$ by $S_{1}$ and the outside of $H_{2}$ is mapped onto the inside of $H_{4}$ by $S_{2}$, we obtain a Fuchsian group $G_{1}$ of the second kind with the fixed circle $|z|=1+\varepsilon_{1}$. Next we describe a circle $H_{5}$ with center at the origin and radius $r=\sqrt{2}-1$ and let it correspond to an elliptic transformation $S_{5}$ with period 2.

Combining Fuchsian group $G_{1}$ with the group $G_{2}$ generated by only $S_{5}$, we obtain a Kleinian group $G$, that is, a combination group $G_{1} \cdot G_{2}$, whose fundamental domain $B_{0}$ is connected and bounded by five circles $H_{j} \quad(j=1,2$, $3,4,5)$.

For convenience of calculation, we may consider the limit case $\varepsilon=0$. In this case $B_{0}$ is no more connected. Then the fixed circle of $G_{1}$ is $|z|=1$. Denote by $D_{j}(j=1,2,3,4,5)$ the closed discs bounded by $H_{j}(j=1,2,3,4,5)$ and $U$ the closed unit disc. The singular set $E$ of $G$ is contained in the domain $\bigcup_{j=1}^{5}\left\{D_{j} \cap U\right\}$. It can be seen from (21) that the generating transformations $S_{j}$ $(j=1,2)$ and $S_{5}$ have the following forms:

$$
\begin{aligned}
& S_{j}=\frac{-\sqrt{2} z+e^{i(2 j-1) \pi / 4}}{e^{-i(2 j-1) \pi / 4} z-\sqrt{2}}, \quad S_{j}^{-1}=S_{j+2}, \quad(j=1,2) \\
& S_{5}=\frac{r^{2} e^{i \theta}}{z}, \quad(\theta ; \quad \forall \text { real number, } r=\sqrt{2}-1) .
\end{aligned}
$$

By virtue of symmetricity of the figure, it is sufficient to calculate the values of the computing functions $f_{s_{5}}^{(2)}(z)$ in $D_{5}$ and $f_{\mathrm{S}_{\mathrm{l}}}^{(2)}(z)$ in $U \cap D_{1}$. Since the centers and radii of the isometric circles $I_{s_{j}}, I_{S_{j}}^{\prime}(j=1,2)$ and $I_{s_{b}}$ are easily known from $(20)$, the values of $f_{S_{1}}^{(2)}(z)$ and $f_{S_{b}}^{(2)}(z)$ in the above restricted domains can be calculated as follows.

(I) Case of $f_{S_{5}}^{(2)}(z)$. 
It holds that in $D_{5}$

$$
f_{S_{\mathrm{s}}}^{(2)}(z)=\sum_{j=1}^{4} \frac{1}{\left|z-g_{j}\right|^{2}},
$$

where $g_{j}$ coincides with the center $a_{j}$ of $H_{j}$. Since $\left|z-g_{j}\right| \leqq 2 \sqrt{2}-1$ in $D_{5}$, we have

$$
f_{B_{b}}^{(2)}(z)>\frac{4}{(2 \sqrt{2}-1)^{2}}=\frac{4(9+4 \sqrt{2})}{49}>1 .
$$

(II) Case of $f_{S_{1}}^{(2)}(z)$.

It holds that in $U \cap D_{1}$

$$
f_{S_{1}}^{(2)}(z)=\sum_{j=2}^{4} \frac{1}{\left|z-g_{j}\right|^{2}}+\frac{(\sqrt{2}-1)^{2}}{|z|^{2}} .
$$

Considering

$$
\frac{1}{\left|z-g_{2}\right|^{2}}+\frac{1}{\left|z-g_{1}\right|^{2}}
$$

we see easily that it attains the minimum at $c_{1}=(1+i) / \sqrt{2}$ in $U \cap U_{1}$. Since $\left|c_{1}-g_{4}\right|^{2}=(\sqrt{2})^{2}+1=3$, the above sum is equal or greater than $2 / 3$. Since $1 /\left|z-g_{3}\right|^{2}$ and $(\sqrt{2}-1)^{2} /|z|^{2}$ attain also the minimum at $c_{1}$, it holds

$$
f_{S_{1}}^{(2)}(z) \geqq \frac{2}{3}+2(\sqrt{2}-1)^{2}>1 .
$$

So taking $\varepsilon$ sufficiently small, we conclude that in this example all functions $f_{s_{j}}^{(2)}(z)(j=1,2,3,4,5)$ are greater than 1 in the singular set contained in $D_{j}$ respectively. Hence for the case of $\mu=2$ the condition of Theorem 1 is satisfied and the 1-dimensional measure of the singular set of such a Kleinian group is positive. Thus the $(-2)$-dimensional Poincaré theta-series $\Theta_{2}(z)$ does not converge in $D^{*}$ for such group.

Remark. We can easily see that for sufficiently small $\delta$ the $(1+\delta)$-dimensional measure of the singular set of the above Kleinian group is positive. Even in the case of $N=5$, there exist Kleinian groups whose singular sets do not belong to Painlevé null sets.

By Example we obtain the following theorem.

THEOREM 2. Under Kleinian groups whose fundamental domains are bounded by mutually disjoint $N(\geqq 5)$ circles, there exist ones with respect to which the 
( -2)-dimensional Poincare theta-series $\Theta_{2}(z)$ does not converge in $D^{*}$ and whose singular sets have positve 1-dimensional measure.

13. Let us consider an application of Theorems 1 and 2 to Schottky groups.

Consider the totality $G^{*}$ formed by the elements of even grade of a Kleinian group $G$. We can easily prove that $G^{*}$ is a Schottky group independent of the sort of the generators.

Take any generator $S_{i_{0}}$ of $G$. Then any element $S_{i_{2}} \cdot S_{i_{1}}$ of grade 2 of $G$ is represented by the form

$$
S_{i_{2}} S_{i_{0}}^{-1} \cdot S_{i_{0}} S_{i_{1}} \text {. }
$$

Therefore any element of $G^{*}$ is generated by

$$
T_{i}=S_{i_{0}} S_{i}, \quad T_{i}^{-1}=\left(S_{i}\right)^{-1} S_{i_{0}}^{-1}=\left(S_{i_{0}} \cdot S_{i}\right)^{-1},
$$

where $S_{i}$ runs in $N-1$ generators except $S_{i_{0}}^{-1}$. We see that $G^{*}$ is a subgroup of $G$ generated by $2 N-2$ generators and their inverses (21). Since $S_{i_{0}}$ is any generator, there are $N$ ways about the determination of the generators of $G^{*}$. Though the generators of $G$ may contain an elliptic transformation with period 2 , the generators of $G^{*}$ are all hyperbolic or loxodromic transformations. Because, since the generator $T_{i}$ maps the boundary circle $K_{i}$ onto a circle of grade 2 in the boundary circle $K_{i_{0}}^{-1}\left(\neq K_{i}\right)$, onto which $S_{i_{0}}$ maps the boundary circle $K_{i_{0}}$, so the circles $K_{i}$ and $T_{i}\left(K_{i}\right)$ are mutually disjoint and hence $T_{i}$ is a hyperbolic or a loxodromic transformation. It is easily seen that the fundamental domain of $G^{*}$ is $B_{0}+S_{i_{0}}\left(B_{0}\right)$. We call such a method, which forms Schottky subgroup from Kleinian group, the inversion method.

14. With respect to a Schottky subgroup $G^{*}$ of $G$ given by inversion method, we have the following

THEOREM 3. If the condition (18) of Theorem 1 is valid for a Kleinian group $G$, then the singular set of a Schottky subgroup $G^{*}$ of $G$ has the positive $\left(\frac{\mu}{2}\right)$ dimensional measure, and the $(x-\mu)$-dimensional $P_{\text {-series }} P_{\mu}(z)$ with respect to $G^{*}$ does not converge in $D^{\prime \prime}$.

Proof. From the assumption of Theorem, it is clear that the singular set $E$ of $G$ has positive $\left(\frac{\mu}{2}\right)$-dimensional measure. Since it can be seen that the singular set $E^{*}$ of $G^{*}$ coincides with $E$ from the definition of the singular set, 
$E^{*}$ has also positive $\left(\frac{\mu}{2}\right)$-dimensional measure.

q.e.d.

15. In Example, take a generator $S_{5}$ and form a system of generators and their inverses :

$$
T_{i}=S_{5} S_{i}, T_{i}^{-1}=S_{i}^{-1} S_{5}^{-1} \quad(i=1,2,3,4) .
$$

Then (22) generate a Schottky subgroup $G^{*}$ of the Kleinian group $G$. Then from Theorems 2 and 3, we obtain the following

Theorem 4. Let $G$ be a Kleinian group whose fundamental domain is bounded by $N$ circles. Under Schottky subgroups given by inversion method from $G$, if $N$ $=5$, there exist Schottky groups whose fundamental domains are bounded by 8 boundary circles and whose singular sets have positive 1-dimensional measure.

16. Problem. The Schottky's condition [7] implies that $l_{m}^{(2)}$ tends to zero for $m \rightarrow \infty$ in Proposition 3. Hence the 1-dimensional measure of the singular set of any Kleinian group with fundamental domain $B_{n}$ bounded by three mutually disjoint circles is always zero, since $B_{0}$ satisfies the Schottky's condition.

Then the 1-dimensional measure of the singular set of a Schottky subgroup with fundamental domain bounded by four circles, which is given from the above Kleinian group by inversion method, is also zero. But it remains to be proved whether there exist or not Kleinian groups with fundamental domains bounded by 4 circles whose singular sets have positive 1-dimensional measure.

\section{REFERENCES}

[1] Akaza, T., Poincaré theta-series and singular sets of Schottky groups. Nagoya Math. Jour. 24 (1964), 43-65.

[2] Ford, L. R., Automorphic Functions. 2nd Ed. Chelsea (1951).

[3] Myrberg, P. J., Zur Theorie der Konvergenz der Poincaréschen Reihen. Ann. Acad. Sci. Fennicae, (A) 9, No. 4 (1916), 1-75.

[4] Myrberg, P. J., Die Kapazität der singulären Menge der linearen Gruppen. Ann. Acad. Sci. Fennicae, A. I. 10 (1941), 1-19.

[5] Petersson, H., Über den Bereich absoluter Konvergenz der Poincaré'schen Reihen. Acta Math. 80 (1948), 23-49.

[6] Sario, L., Über Riemannsche Flächen mit hebbarem Rand. Ann. Acad. Sci. Fennicae, A. E. 50 (1948), 1-79.

[7] Schottky, F., Über eine specialle Function, welche bei einer bestimmten linearen Transformation ihres Arguments unverädert bleibt. Crelle's Jour. 101 (1887), 227-272.

Mathematical Institute

Kanazawa University 